\title{
ÁCAROS (ARACHNIDA: ACARI) ASSOCIADOS A PLANTAS ORNAMENTAIS TROPICAIS NA REGIÃO LITORAL SUL DA BAHIA
}

\author{
R.M.V. Santos ${ }^{1}$, A.C.S. Noronha ${ }^{2}$, A.R. Oliveiraa ${ }^{1}$, M.A.L. Bittencourt ${ }^{1}$ \\ ${ }^{1}$ Universidade Estadual de Santa Cruz, Departamento de Ciências Agrárias e Ambientais, Rodovia Ilhéus - \\ Itabuna, km 16, CEP 45662-000, Ilhéus, BA, Brasil. E-mail: renatavitalagro@gmail.com
}

\section{RESUMO}

Em razão da expansão do agronegócio de flores tropicais na região Litoral Sul da Bahia e da inexistência de estudos com ácaros associados a essas culturas na região foram realizadas coletas no período de setembro de 2006 a junho de 2007 em 34 espécies/cultivares de plantas ornamentais tropicais das famílias Costaceae, Heliconiaceae, Musaceae e Zingiberaceae de onze propriedades localizadas em seis municípios $\left(13^{\circ} 21^{\prime}\right.$ a $14^{\circ} 58^{\prime}$ de latitude Sul e $39^{\circ} 04^{\prime}$ a $39^{\circ} 27^{\prime}$ de longitude Oeste), com o objetivo de se determinar a composição de grupos funcionais nos cultivos analisando-se a comunidade no nível de família por meio de índices faunísticos. Os ácaros foram coletados, mensalmente, em folhas e inflorescências. Foram registradas 22 famílias. Mais da metade dos ácaros pertenceu a famílias predominantemente predadoras, com destaque para os fitoseídeos. Com base em índices faunísticos, as famílias Phytoseiidae e Tetranychidae atingiram os índices máximos de dominância, abundância, frequência e constância. Tetraniquídeos (Tetranychus aff. abacae Baker \& Pritchard) foram registrados em Alpinia purpurata (Viell.) Schum, Etlingera elatior (Jack) R. M. Smith, Musa coccinea H.C. Andrews, Strelitzia reginae Aiton, Zingiber spectabilis Griffith, Heliconia latispatha Benth., Heliconia rivularis L. Emygd. \& E. Santos, Heliconia rostrata Ruiz \& Pavon, Heliconia wagneriana Petersen e nos cultivares de helicônias 'Nappi Yellow', 'Jacquinii', 'She', 'Golden Torch', 'Red Opal', 'Alan Carle', 'Fire Bird' e 'Nappi Red'. As espécies/cultivares com maior número de famílias de ácaros foram $H$. latispatha, H. rostrata, M. coccinea, 'Fire Bird', E. elatior 'Vermelha', Z. spectabilis, E. elatior 'Rosa', A. purpurata 'Vermelha' e helicônias 'Alan Carle', 'Sassy' e 'She'. Não foram detectados nas plantas danos que causassem prejuízos econômicos aos produtores. As pequenas populações de fitófagos sugerem um eficiente controle natural por predadores, patógenos ou outros fatores.

PALAVRAS-CHAVE: Acarofauna, diversidade, Phytoseiidae, Tetranychidae, helicônia.

\begin{abstract}
MITES(ARACHNIDA: ACARI) ASSOCIATEDWITHTROPICALORNAMENTALPLANTSIN THE SOUTHERN COASTAL REGION OF BAHIA, BRAZIL. Due to the expansion of the tropical flower agribusiness in the southern coastal region of Bahia State, Brazil, and the lack of studies on mites associated to these cultures in the region, mite surveys were conducted from September 2006 to June 2007 in 34 species/cultivars of ornamental plants of the families Costaceae, Heliconiaceae, Musaceae and Zingiberaceae on 11 properties located in 6 counties $\left(13^{\circ} 21^{\prime}\right.$ to $14^{\circ} 58^{\prime}$ of south latitude and $39^{\circ} 04^{\prime}$ to $39^{\circ} 27^{\prime}$ of west longitude) with the aim of determining the composition of functional groups in the field through the analysis of the community at the family level using faunistic indexes. Mites were collected monthly from leaves and inflorescences. Twenty-two families were recorded. More than half of the mites pertained to predominantly predatory families, mainly to phytoseiids. Based on faunistic indexes, the families Phytoseiidae and Tetranychidae showed the maximum indexes of dominance, abundance, frequency and constancy. Tetranychids (Tetranychus aff. abacae Baker \& Pritchard) were reported on Alpinia purpurata (Viell.) Schum, Etlingera elatior (Jack) R. M. Smith, Musa coccinea H.C. Andrews, Strelitzia reginae Aiton, Zingiber spectabilis Griffith, Heliconia latispatha Benth., Heliconia rivularis L. Emygd. \& E. Santos, Heliconia rostrata Ruiz \& Pavon, Heliconia wagneriana Petersen and on the cultivars of heliconia 'Nappi Yellow,' 'Jacquinii,' 'She,' 'Golden Torch,' ‘Red Opal,' 'Alan Carle,' ‘Fire Bird' and 'Nappi Red.' The species/cultivars with the largest number of mite families were H. latispatha, H. rostrata, M. coccinea, 'Fire Bird,' E. elatior 'Vermelha,' Z. spectabilis, E. elatior 'Rosa,' A. purpurata 'Vermelha' and heliconias 'Alan
\end{abstract}

${ }^{2}$ Embrapa Amazônia Oriental, Belém, PA, Brasil. 
Carle,' 'Sassy' and 'She.' No mite-induced economic losses on tropical flower cultures were observed. The low population levels of phytophagous mites suggest an efficient natural control by predators, pathogens and other factors.

KEY WORDS: Mite fauna, diversity, Phytoseiidae, Tetranychidae, heliconia.

\section{INTRODUÇÃO}

O mercado mundial de flores tropicais apresenta elevado potencial de crescimento (CASTRO et al., 2007), sendo que as exportações brasileiras de flores e plantas ornamentais cresceram mais de $124 \%$ entre 2001 e 2006, mantendo um crescimento real de pelo menos $10 \%$ ao ano (JUNQUeIRA; PeETZ, 2007). O cultivo de flores tropicais no Brasil tem se destacado principalmente nos estados do Nordeste, com importantes reflexos sócio-econômicos para a região (Assis et al., 2002; JunQueIRA; PeETZ, 2005, 2007), e a Bahia, especialmente na região Litoral Sul, vem incrementando o comércio de flores tropicais, com uma produção estimada de cerca de 300 mil dúzias de flores tropicais e subtropicais por ano (SCHERER, 2006; BAHIA, 2007).

Nas famílias Araceae, Heliconiaceae, Musaceae e Zingiberaceae encontram-se as espécies mais importantes da floricultura tropical, com destaque para as helicônias, muito apreciadas tanto no mercado interno quanto externo, pela durabilidade, beleza e variedade de formas e cores (Assis et al., 2002; VALLESPIR, 2005). No entanto, a grande expansão observada recentemente das áreas de cultivo de helicônias no país tem implicado na elevação dos problemas fitossanitários (Assis et al., 2002).

O ácaro-vermelho Tetranychus abacae Baker \& Pritchard (Tetranychidae) ocorre no Brasil, Colômbia, Costa Rica, Honduras, Porto Rico e Venezuela em hospedeiros das famílias Heliconiaceae e Musaceae, tendo sido registrados danos desse ácaro em diferentes espécies de helicônias no Sudeste e no Nordeste do Brasil (MoraEs; Flechtmann, 1981; Flechtmann, 1996; Assis et al., 2002; VASCONCELOSet al., 2004; MoRAEs; FleCHTMANN, 2008). As populações de T. abacae se desenvolvem, principalmente, na face inferior das folhas, que ficam recobertas com grande quantidade de teia. Os danos iniciam com o aparecimento de clorose, progredindo para secamento e queda prematura de folhas, principalmente durante estações mais secas do ano (Assis et al., 2002; VAsCONCELOS et al., 2004; Moraes; Flechtmann, 2008).

Apesar da expansão dos cultivos de plantas ornamentais tropicais na região Litoral Sul da Bahia, inexistem estudos com ácaros associados a estas culturas. Considerando-se que o conhecimento sobre os padrões de ocorrência e de diversidade em comunidades deácaros associados a plantas em uma dada região é uma etapa básica e imprescindível na elaboração de propostas de manejo deespécies pragas (FERES et al., 2003), analisou-se no presente trabalho a composição de grupos funcionais (fitófagos, predadores e outros) prevalecente em cultivos dessas plantas na região analisando-se a comunidade no nível de família por meio de índices faunísticos, que é uma técnica utilizada para caracterizar e delimitar uma comunidade, medir o impacto ambiental de ações antrópicas em uma área, e conhecer as espécies e famílias predominantes (Silveira Neto et al., 1976).

\section{MATERIAL E MÉTODOS}

O trabalho foi realizado de setembro de 2006 a junho de 2007 em áreas cultivadas com plantas ornamentais tropicais produtoras de flores das famílias Costaceae, Heliconiaceae, Musaceaee Zingiberaceae de onze propriedades localizadas nos municípios de Ibirapitanga, Ilhéus, Itabuna, Ituberá, Uruçuca e Valença $\left(13^{\circ} 21^{\prime}\right.$ a $14^{\circ} 58^{\prime}$ de latitude Sul e $39^{\circ} 04^{\prime}$ a $39^{\circ} 27^{\prime}$ de longitudeOeste). As34 espécies/cultivares amostradas foram: Alpinia purpurata (Viell.) Schum 'Rosa' e'Vermelha', Etlingeraelatior (Jack) R. M.Smith 'Porcelana', 'Rosa' e 'Vermelha', Heliconia bihai (L.) L., H. bihai 'Nappi Red' e 'Nappi Yellow', Heliconia caribaea Lamarck x Heliconia bihai (L.) L. 'Dimitri Sucre' e 'Jacquinii', Heliconia latispatha Benth., Heliconia ortotricha Candy Cane 'She' e 'Total Eclipse', Heliconia pendula Wawra 'Bright Red', H. psittacorum L. 'Adromeda', 'Golden Torch', 'Lady Di', 'Red Opal' e 'Sassy', H. psittacorum x Heliconia marginata (Griggs) Pittier 'Nickeriensis', H. psittacorum x Heliconia spathocircinata Aristeg. 'Alan Carle' e 'Golden Torch Adrian', Heliconia rauliniana Barreiros, Heliconia rivularis L. Emygd. \& E. Santos, Heliconia rostrata Ruiz \& Pavon, H. rostrata 'Pilosa', Heliconia sarapiquensis G.S. Daniels \& F.G. Stiles, Heliconia stricta Huber 'Fire Bird' e 'Xingu', Heliconia wagneriana Petersen, Musa coccinea H.C. Andrews, Strelitzia reginae Aiton, Tapeinochilos ananassae (Hassk.) K. Schum., Zingiber spectabilis Griffith. A identificação das espécies/ cultivares foi baseada no relato dos produtores (aquisição das mudas) e em LAmAs (2004).

Mensalmente, foram amostradas aleatoriamente três folhas (basal, intermediária e apical) e uma inflorescência por planta em dez espécies/cultivares 
vegetais por propriedade. As amostras foram embaladas em sacos de papel, etiquetadas, acondicionadas em sacos plásticos e transportadas ao laboratório. As partes vegetais foram mantidas em refrigerador por, no máximo, sete dias, quando as amostras foram examinadas sob microscópio estereoscópico, sendo todos os ácaros coletados com um pincel de cerdas finas e transferidos para microtubos ('Eppendorf') com álcool etílico (70\%). Para identificação, os espécimes foram montados (exceto adultos de Oribatida) em lâminas de microscopia em meio de Hoyer, segundo a metodologia recomendada por FLECHTmanN (1975), Krantz (1978) e Moraes; Flechtmann (2008). Os Oribatida adultos foram examinados em lâmina escavada contendo uma gota de ácido lático, parcialmente coberta com uma lamínula (TRAVÉ et al., 1996; Oliveira, 2004).

A identificação das famílias foi realizada com auxílio de um microscópio fotônico de contraste de fases, com base em FLECHTMANN $(1975,1985)$, KRANTZ (1978), Balogh; Balogh (1992) e Moraes; FlechtMAnN (2008). As famílias foram classificadas de acordo com Moraes; Flechtmann (2008) e Subías (2004) e o hábito alimentar generalizado de cada grupo definido com base em FlechtMann (1975, 1985), KRANTZ (1978) e Moraes; Flechtmann (2008), a fim de se determinar a composição de grupos funcionais (fitófagos, predadores e outros) nos cultivos.

Cada família de ácaros foi categorizada quanto à sua dominância, abundância, frequência econstância na comunidade (Silveira Neto et al., 1976; URAMOto et al., 2005). Os índices faunísticos foram calculados com base no programa ANAFAU (MORAES et al., 2003) desenvolvido no Departamento de Entomologia, Fitopatologia e Zoologia Agrícola da Escola Superior de Agricultura Luiz de Queiroz/USP, em Piracicaba, SP.

Os índices de pluviosidade para todos os municípios de coleta foram obtidos junto ao Ministério da Agricultura, Pecuária e Abastecimento, por meio do Centro de Pesquisas do Cacau/Comissão Executiva do Plano da Lavoura Cacaueira (CEPEC/CEPLAC), no Município de Ilhéus, Bahia.

\section{RESULTADOS}

Duzentos e oitenta e três ácaros em quatro ordens e 22 famílias foram determinados (Tabela 1). Quanto ao hábito alimentar generalizado, Eriophyidae, Tarsonemidae, Tenuipalpidae e Tetranychidae foram classificados como fitófagos; Ascidae, Cunaxidae, Macrochelidae, Phytoseiidae e Stigmaeidae como predominantemente predadores eas demaisfamílias como representantes de outros hábitos alimentares: micófagos, saprófagos e outros.

Considerando-se todas as coletas e localidades, os Mesostigmata (46\%) prevaleceram em relação aos Prostigmata (34\%), Oribatida (15\%) e Astigmata $(5 \%)$. Apenas $20 \%$ dos ácaros pertenceram às famílias fitófagas, $29 \%$ às famílias com outros hábitos alimentares e mais da metade (51\%) às famílias predominantemente predadoras, sendo que os Phytoseiidae representaram $36 \%$ de todos os ácaros coletados nesse estudo. A maioria dos fitoseídeos foi coletada nas folhas, sendo apenas dois exemplares obtidos nas flores.

Em relação aos índices faunísticos (Tabela 1), as famílias Ascidae, Eupodidae, Phytoseiidae e Tetranychidae foram consideradas dominantes, constantes e muito frequentes. Tetranychidae e Phytoseiidae, por sua vez, foram classificadas como muito abundantes, Ascidae e Eupodidae como abundantes, Acaridae, Cunaxidae, Haplozetidae, Scheloribatidae e Tenuipalpidae como comuns, e as demais como dispersas ou raras.

Foi registrada uma alta diversidade nas áreas de cultivos, como ficou demonstrado pelos valores dos índices de Shannon-Weaner $(2,3374)$ e de equitabilidade $(0,7455)$ (Tabela 1$)$. As famílias que predominaram nos cultivos foram Phytoseiidae e Tetranychidae, que atingiram os índices máximos de dominância, abundância, frequência e constância. Além de terem sido as únicas famílias registradas em todos os municípios, fitoseídeos e tetraniquídeos, juntos, representaram mais de $50 \%$ de todos os ácaros coletados, ocorrendo na maioria das espécies/cultivares vegetais. Os Phytoseiidae ocorreram em 25, os Tetranychidae em 19 e as demais famílias em no máximo 10 das 34 espécies/cultivares amostradas. Os Tetranychidae (provavelmente T. abacae) foram encontrados nas seguintes espécies/cultivares: $A$. purpurata 'Vermelha'; E. elatior 'Porcelana', 'Rosa' e 'Vermelha'; M. coccinea; S. reginae; Z. spectabilis; $H$. latispatha, $H$. rivularis, $H$. rostrata, $H$. wagneriana e nos cultivares 'Nappi Yellow', 'Jacquinii', 'She', 'Golden Torch', 'Red Opal', 'Alan Carle', 'Fire Bird' e 'Nappi Red'. Em apenas 'Nappi Yellow' e'Jacquinii' não foram encontrados fitoseídeos associados aos tetraniquídeos.

As espécies/cultivares de plantas que apresentaram maior número de famílias de ácaros foram: H. latispatha (12 famílias), H. rostrata (11), M. coccinea (10), 'Fire bird' (9), E. elatior 'Vermelha' (8), Z. spectabilis (7), E. elatior 'Rosa' e A. purpurata 'Vermelha' (6), 'Alan Carle', 'Sassy' e 'She' (5). Não foram detectados danos nas espécies vegetais coletadas que causassem prejuízos econômicos aos produtores da região Litoral Sul da Bahia.

O total acumulado de precipitação nos dez meses de coleta variou de $1.323 \mathrm{~mm}$ em Itabuna a 1965 $\mathrm{mm}$ em Valença, com chuvas distribuídas ao longo do período e maior índice de precipitação no mês de fevereiro. 
Tabela 1 - Classes de dominância, abundância, frequência e constância das famílias de ácaros coletados em cultivos de plantas ornamentais tropicais produtoras de flores de seis municípios da região Litoral Sul da Bahia, de setembro de 2006 a junho de 2007.

\begin{tabular}{|c|c|c|c|c|c|}
\hline Ordem/Família & Total ${ }^{(1)}$ & Dominância ${ }^{(2)}$ & Abundância ${ }^{(3)}$ & Frequência ${ }^{(4)}$ & Constância $^{(5)}$ \\
\hline \multicolumn{6}{|l|}{ Astigmata } \\
\hline Acaridae & 14 & $\mathrm{D}$ & c & $\mathrm{F}$ & $\mathrm{Y}$ \\
\hline \multicolumn{6}{|l|}{ Mesostigmata } \\
\hline Macrochelidae & 4 & ND & $\mathrm{d}$ & PF & Z \\
\hline Ascidae & 24 & $\mathrm{D}$ & a & MF & W \\
\hline Phytoseiidae & 101 & $\mathrm{D}$ & ma & MF & W \\
\hline \multicolumn{6}{|l|}{ Prostigmata } \\
\hline Cunaxidae & 12 & $\mathrm{D}$ & c & $\mathrm{F}$ & $\mathrm{Y}$ \\
\hline Eriophyidae & 2 & ND & $\mathrm{d}$ & PF & Z \\
\hline Eupodidae & 23 & $\mathrm{D}$ & $\mathrm{a}$ & MF & W \\
\hline Stigmaeidae & 3 & ND & $\mathrm{d}$ & PF & $\mathrm{Z}$ \\
\hline Tarsonemidae & 3 & ND & $\mathrm{d}$ & PF & $\mathrm{Z}$ \\
\hline Tenuipalpidae & 9 & $\mathrm{D}$ & c & $\mathrm{F}$ & $\mathrm{Y}$ \\
\hline Tetranychidae & 44 & $\mathrm{D}$ & ma & $\mathrm{MF}$ & W \\
\hline Tydeidae & 1 & ND & $\mathrm{r}$ & PF & $\mathrm{Z}$ \\
\hline \multicolumn{6}{|l|}{ Oribatida } \\
\hline Austrachipteriidae & 4 & ND & $\mathrm{d}$ & PF & Z \\
\hline Ceratokalummidae & 1 & ND & $\mathrm{r}$ & PF & $\mathrm{Z}$ \\
\hline Galumnidae & 2 & ND & $\mathrm{d}$ & PF & Z \\
\hline Haplozetidae & 15 & $\mathrm{D}$ & c & $\mathrm{F}$ & $\mathrm{W}$ \\
\hline Hemileiidae & 1 & ND & $\mathrm{r}$ & PF & $\mathrm{Z}$ \\
\hline Mochlozetidae & 5 & ND & $\mathrm{d}$ & PF & Z \\
\hline Oppiidae & 3 & ND & $\mathrm{d}$ & PF & Z \\
\hline Oribatulidae & 1 & ND & $\mathrm{r}$ & PF & $\mathrm{Z}$ \\
\hline Scheloribatidae & 10 & $\mathrm{D}$ & c & $\mathrm{F}$ & $\mathrm{Y}$ \\
\hline Scutoverticidae & 1 & ND & $\mathrm{r}$ & PF & Z \\
\hline
\end{tabular}

Índice de Shannon-Weaner $=2,3374$

Intervalo de confiança $(\mathrm{P}=0,005)=[2,330239 ; 2,344545]$

Índice de uniformidade ou equitabilidade $=0,7455$

(1) Total de espécimes coletados;

(2) $\mathrm{D}=$ dominante; ND = não dominante;

(3) $\mathrm{ma}=$ muito abundante; $\mathrm{a}=$ abundante; $\mathrm{c}=$ comum; $\mathrm{d}=$ dispersa; $\mathrm{r}=$ rara;

(4) $\mathrm{MF}=$ muito frequente; $\mathrm{F}=$ frequente; $\mathrm{PF}=$ pouco frequente;

(5) $\mathrm{W}=$ constante; $\mathrm{Y}=$ acessória; $\mathrm{Z}=$ acidental.

\section{DISCUSSÃO}

Em relação ao total deácaros coletados, observouse que houve predominância dos Phytoseiidae em relação às demais famílias. Além de apresentarem hábito predatório, muitas espécies também podem utilizar outras fontes dealimento como pólen, fungos, exsudatos de plantas e secreções adocicadas, o que propicia a manutenção de suas populações nas áreas decultivo mesmo quando há pequena quantidade de presas (Jeprson et al., 1975; McMurtry; Croft, 1997; MORAes; FleChTMANN, 2008). Predadores generalistas como Ascidae e Cunaxidae poderiam também estar contribuindo para a baixa ocorrência de fitófagos observada neste estudo (MORAES; FLECHTMANN, 2008).
Os Tetranychidae representaram 15\% do total de ácaros coletados. Estes ácaros ocorrem em grande número de hospedeiros e algumas espécies apresentam importânciaeconômicano Brasil(MORAES; FLECHTMANN, 2008). Tetranychus abacae é a única espécie conhecida de Tetranychidae encontrada associada Heliconiaceae e Musaceae (FlechtmanN, 1996; Assis et al., 2002). VASCONCELOS et al. (2004) estimaram que podem ocorrer 31 gerações por ano dessa espécie a $26^{\circ} \mathrm{C} \mathrm{em}$ Musa sp. cv. Prata, ressaltando que a expansão do cultivo de flores tropicais pode afetar o equilíbrio do ácaro, tornando-o uma praga potencial no cultivo de helicônias, principalmenteem cultivo protegido. $\mathrm{Na}$ região Litoral Sul da Bahia, embora T. abacae ainda não tenha sido registrado, a existência de grandes 
áreas cultivadas com bananeira e a ampliação dos cultivos de flores tropicais poderia favorecer a presença de grandes populações dessa espécie.

O alimento, como um componente do ambiente, influi diretamente na distribuição e abundância dos artrópodes, afetando os processos biológicos e comportamentais (SilveiRa Neto et al., 1976), de modo que os predadores generalistas têm maior possibilidade de distribuição. Considerando-se que apenas $20 \%$ do total de espécimes coletados pertenceram a famílias fitófagas (Eriophyidae, Tarsonemidae, Tenuipalpidae e Tetranychidae) e que os Phytoseiidae corresponderam a $36 \%$, pode-se sugerir ser essa uma consequência do comportamento alimentar genera-lista da maioria das espécies de fitoseídeos, além do clima (úmido) da região, que poderiam estar controlando as populações de fitófagos.

Vários fatores podem ter influenciado as pequenas populações de fitófagos nos cultivos amostrados, tais como a ação de inimigos naturais (ácaros e insetos predadorese/ou fungosentomopatogênicos), favorecidos pela diversidade de cultivos e de áreas com vegetação natural comuns na região, servindo como áreas de refúgio (Altieri et al., 2003). Também as condições climáticas, particularmente as chuvas, abundantes na região no período de coleta, pela ação mecânica direta sobre os ácaros e elevada umidade relativa do ar, podem ter contribuído. Os Tetranychidae, grupo predominante de fitófagos registrado neste trabalho, são favorecidos por baixos níveis de umidade no ar e baixos níveis de precipitação (VIEIRA et al., 2004).

Embora não tenham sido coletados machos de Tetranychidae, estes foram afiliados ao gênero Tetranychus Dufour e todas as características das fêmeas sugerem que os exemplares são de T. abacae. Apesar dos tetraniquídeos terem sido considerados predominantes por meio dos índices faunísticos e coletados em mais da metade das espécies/cultivares de plantas ornamentais tropicais amostradas, a ausência de danos pode ser atribuída às suas pequenas populações.

Este trabalho apresenta as primeiras informações sobre a acarofauna em cultivos de flores tropicais na Bahia, contribuindo para o conhecimento da composição de grupos de ácaros, principalmente fitófagos e predadores, associados a plantas das famílias Costaceae, Heliconiaceae, Musaceaee Zingiberaceae para a região Litoral Sul. A determinação no nível de espécie dos grupos de fitófagos, predadores e outros, as funções desempenhadas no agroecossistema, assim como as interações com fatores bióticos e abióticos merecem ser estudadas.

\section{CONCLUSÕES}

Phytoseiidae e Tetranychidae são dominantes, abundantes, frequentes e constantes em plantas ornamentais tropicais produtoras de flores na região Litoral Sul da Bahia. As espécies/cultivares com maior número de famílias de ácaros foram H. latispatha, $H$. rostrata, M. coccinea, 'Fire bird', E. elatior 'Vermelha', Z. spectabilis, E. elatior 'Rosa' e A. purpurata 'Vermelha', 'Alan Carle', 'Sassy' e 'She'. Não foram detectados danos nas espécies vegetais coletadas que causassem prejuízos econômicos aos produtores da região Litoral Sul da Bahia.

\section{AGRADECIMENTOS}

À Fundação de Amparo à Pesquisa do Estado da Bahia(FAPESB) pelas bolsas de mestradoe PRODOC concedidas ao primeiro e terceiro autores, respectivamente, e À Universidade Estadual de Santa Cruz (UESC) onde foi realizado o trabalho. Aos produtores de flores tropicais, que gentilmente permitiram a realização das coletas em seus cultivos.

\section{REFERÊNCIAS}

ALTIERI, M.A.; SILVA, E.M.; NICHOLLS, C.I. O papel da biodiversidade no manejo de pragas. Ribeirão Preto: Holos, 2003. 226p.

ASSIS, S.M.P.; MARIANO, R.R.L.; GONDIM JUNIOR, M.G.C.; MENEZES, M.; ROSA, R.C.T. da Doenças e pragas das helicônias (Diseases and pests of heliconias). Recife: UFRPE, 2002. 102p.

BAHIA. Secretaria da Agricultura, Irrigação e Reforma Agrária. Projeto "Flores da Bahia", 2007. Disponível em: <http://www. seagri.ba. gov.br/programas.asp?qact= viewprogram\&prgid $=18>$. Acesso em: 30 set. 2008.

BALOGH, J.; BALOGH, P. The oribatid mites genera of the world. Budapest: Hungarian Natural History Museum, 1992. 2v, 638p.

CASTRO, A.C.R. de; LOGES, V.; COSTA, A.S. da; CASTRO, M.F.A. de; ARAGÃO, F.A.S. de; WILLADINO, L.G. Hastes florais de helicônia sob deficiência de macronutrientes. Pesquisa Agropecuária Brasileira, v.42, n.9, p.1299-1306, 2007.

FERES, R.J.F.; BELLINI, M.R.; ROSSA-FERES, D. de C. Ocorrência e diversidade de ácaros (Acari, Arachnida) associados a Tabebuia roseo-alba (Ridl.) Sand (Bignoniaceae), no município de São José do Rio Preto, São Paulo, Brasil. Revista Brasileira de Zoologia, v.20, n.3, p.373-378, 2003.

FLECHTMANN, C.H.W. Elementos de acarologia. São Paulo: Nobel, 1975. 344p.

FLECHTMANN, C.H.W. Ácaros de importância agrícola. São Paulo: Livraria Nobel, 1985. 189p. 
FLECHTMANN, C.H.W. Rediscovery of Tetranychus abacae Baker \& Pritchard, additional description and notes on South American spider mites (Acari, Prostigmata, Tetranychidae). Revista Brasileira de Zoologia, v.13, n.3, p.569-578, 1996.

JEPPSON, L.R.; KEIFER, H.H.; BAKER, E.W. Mites injurious to economic plants. Berkeley: University of California, 1975. 614p.

JUNQUEIRA, A.H.; PEETZ, M.S. Comercialização: aspectos de mercado e manuseio pós-colheita. In: TERAO, D.; CARVALHO, A.C.P.P.; BARROSO, T.C.S.F. (Ed.). Flores tropicais. Brasília: Embrapa Informação Tecnológica/Fortaleza: Embrapa Agroindústria Tropical, 2005. p.73-181.

JUNQUEIRA, A.H.; PEETZ, M.S. Las exportaciones brasileñas de flores y plantas ornamentales crecen más del $124 \%$ entre 2001 y 2006. Horticultura Internacional, n.56, p.76-78, 2007.

KRANTZ, G.W. A manual of acarology. 2.ed. Corvallis: Oregon State University, 1978. 509p

LAMAS, A.M. Floricultura tropical: tecnologia de cultivo. Maceió: SEBRAE, 2004. 65p. [Apostila].

McMURTRY, J.A.; CROFT, B.A. Life-styles of phytoseiid mites and their roles in biological control. Annual Review of Entomology, v.42, p.291-321, 1997.

MORAES, G.J. de; FLECHTMANN, C.H.W. Ácaros fitófagos do Nordeste do Brasil. Pesquisa Agropecuária Brasileira, v.16, n.2, p.177-186, 1981.

MORAES, G.J. de; FLECHTMANN, C.H.W. Manual de Acarologia: acarologia básica e ácaros de plantas cultivadas no Brasil. Ribeirão Preto: Holos, 2008. $288 \mathrm{p}$.

MORAES, R.C.B.; HADDAD, M.L.; SILVEIRA NETO, S.; REYES, A.E.L. Software para análise estatística - ANAFAU. In: SIMPÓSIO DE CONTROLE BIOLÓGICO, 8., 2003, São Pedro, SP. Resumos. Piracicaba: ESALQ/USP, 2003. p.195.
OLIVEIRA, A.R. Diversidade de ácaros oribatídeos (Acari: Oribatida) edáficos e plantícolas do Estado de São Paulo. 2004. 186p. Tese (Doutorado) - Instituto de Biociências, Universidade de São Paulo, São Paulo, 2004.

SCHERER, A.M.S. As flores da Bahia. Bahia Agrícola, v.7, n.3, p.9-13, 2006.

SILVEIRA NETO, S.; NAKANO, O.; BARBIN, D.; VILANOVA, N.A. Manual de ecologia dos insetos. São Paulo: Agronômica Ceres, 1976. 419p.

SUBÍAS, L.S. Listado sistemático, sinonímico y biogeográfico de los ácaros oribáticos (Acariformes, Oribatida) del mundo (1758-2002). Graellsia, v.60, p.1-305, 2004. Número extraordinário.

TRAVÉ, J.; ANDRÉ, H.M.; TABERLY, G.; BERNINI, F. Les acariens oribates. Wavre: AGAR/SIALF, 1996. 110p. (Études en Acarologie, 1).

URAMOTO, K.; WALDER, J.M.M.; ZUCCHI, R.A. Análise quantitativa e distribuição de populações de espécies de Anastrepha (Diptera: Tephritidae) no Campus Luiz de Queiroz, Piracicaba, SP. Neotropical Entomology, v.34, n.1, p.33-39, 2005.

VALLESPIR, A.N. Comércio ornamental: Brasil y el mercado mundial. Horticultura Internacional, n.47, p.1226, 2005.

VASCONCELOS, G.J.N.; SILVA, F.R. da; GONDIM JUNIOR, M.G.C.; BARROS, R.; OLIVEIRA, J.V. Efeito de diferentes temperaturas no desenvolvimento e reprodução de Tetranychus abacae Baker \& Printchard (Acari: Tetranychidae) em bananeira Musa sp. cv. Prata. Neotropical Entomology, v.33, n.2, p.149-154, 2004.

VIEIRA, M.R.L.C.; CASTRO, T.M.M.G.; SILVA, L.F.S.; MONTEVERDE, M.S. Efeito do cultivo do mamoeiro (Carica papaya L.) em ambiente protegido sobre a ocorrência de ácaros fitófagos e moscas-brancas. Revista Brasileira de Fruticultura, v.26, n.3, p.441-445, 2004

Recebido em $7 / 10 / 08$

Aceito em 9/10/09 